\title{
Functional Evolution of Software: FOCUs Model of Design Rationale
}

\author{
Irene Au \\ Netscape Communications Corporation \\ Mountain View, CA 94043 \\ i-au@netscape.com
}

\author{
Penelope Sanderson \\ SCHIL, School of Information Technology \\ Hawthorn, VIC 3122 \\ Swinburne University of Technology \\ psanderson@swin.edu.au
}

\begin{abstract}
In this poster we present the FOCUs model for representing design rationale. The FOCUs model assimilates the benefits of the MacLean, Young, Bellotti, and Moran's (1996) QOC model and the interdisciplinary constraints and pressures represented in Rasmussen, Pejtersen, and Goodstein's (1994) domain maps. A set of FOCUs maps for a particular design problem can be generated and organized into a lattice to represent design rationale from the most general to most specific detail. We have applied the FOCUs model to the development of a smart scheduling feature in two different but related software applications. We argue that the FOCUs model is particularly useful for tracking the evolution of functional prototypes and concept demonstrators.
\end{abstract}

KEYWORDS. Design rationale, software engineering, design space analysis, QOC model, interdisciplinary design

\section{DESIGN RATIONALE}

Software design and development is a dynamic process that involves forging a path from an initial need to a fully functional and usable product. HCI researchers have been seeking ways of better understanding, facilitating, and documenting this process (Moran \& Carroll, 1996). In this poster we present a new model-FOCUs (Au, 1996)-developed to capture the design rationale invoked when we added a smart scheduling feature to the Advising Workbench (AWB) software for academic advising at University of Illinois (Sanderson, Iozzo, Buberel, \& Au, 1995).

The smart scheduling feature takes a student's proposed list of courses and, from the multiple sections offered within each course, finds many possible weekly schedules to choose from. Because the design process for the smart scheduler was complicated by multiple constraints, we performed a post-hoc analysis of design rationale using MacLean, Young, Bellotti, and Moran's (1996) Questions-Options-Criteria (QOC) framework and Rasmussen, Pejtersen, and Goodstein's (1994) domain maps. The QOC notation successfully represented some of the design rationale for the smart scheduler but it had no compelling way of representing the effects of criteria imposed on the design by different constituencies involved in the product. In contrast,
Rasmussen et al.'s (1994) domain maps showed how design is molded by the competing needs of different constituencies, but assumptions made about the problem, the tradeoffs considered between options, and the compromises made, were not reflected. Moreover, transitions between the domain map cells only represented temporal sequence, which did not express implications or design interactions that occur in parallel.

\section{FOCUS MODEL}

To cover the above inadequacies we developed the FOCUs model of design rationale (see Figure 1). FOCUs (Features, Organizational support, Computer science, and Users) is a descriptive model of software evolution and is a symbiosis between the QOC notation and Rasmussen's domain maps (Au, 1996).

Like the latter, FOCUs maps use a grid to lay out the design process (Fifure 1) Rows represent the features wanted $(F)$, the organizational support available to provide access to resources during development $(\mathrm{O})$, computational issues (C), and usability issues (Us). Columns represent the Goals $(G)$ of the different interests represented, Requirements or Rationale $(\mathrm{Rq})$, Constraints $(\mathrm{Cn})$ imposed by some interest, and Resolution ( $\mathrm{Re})$. 


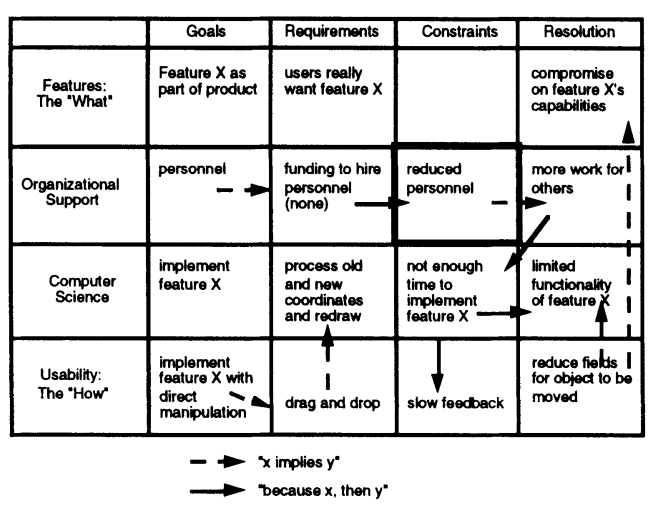

Figure 1: A sample FOCUs map from a simple design problem (not smart scheduling).

Because design resolutions may be linked or nested within each other, such relations must be shown. FOCUs maps can be organized into a lattice (see Figure 2) so that specific solutions to more general problems are visualized appropriately.

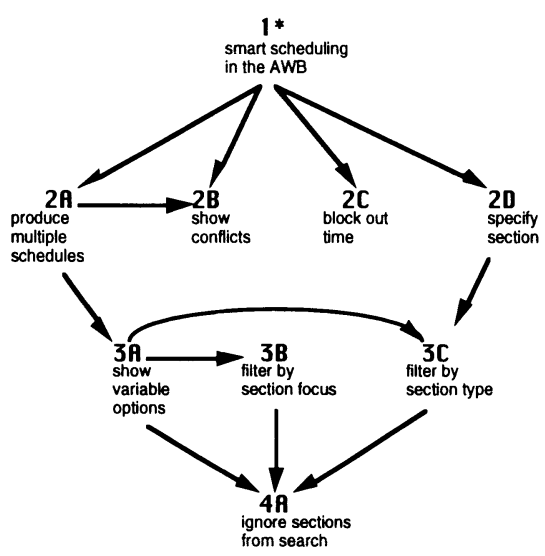

Figure 2: Lattice of nine FOCUs maps detailing design rationale for the smart scheduling feature of the AWB.

Overall, we found that the FOCUs maps throw into sharp relief the sources of compromise that shaped the current form of the smart scheduler. They illustrated the pervasive effect of particular constraints and helped us prioritize efforts to remove these constraints, some of which involved significant organizational negotiation.

\section{EVALUATION OF FOCUS MODEL}

For the smart scheduling prototyping problem, the FOCUs approach inherits the advantages but avoids some disadvantages of the QOC and Rasmussen approaches. (1) The lattice of FOCUs maps allows different levels of detail to be expressed. (2) FOCUs maps make it easy to see the constraints imposed on the design by the different domains and constituencies involved. (3) FOCUs maps are both descriptive and explanatory, revealing how the software evolved and why. (4) FOCUs maps are sensitive to differences in implementation, tradeoffs, and compromises. (5) The arrows linking cells entries allow developers to visualize the complexity of the design problem and to predict potential problems if requirements or constraints change. (6) A set of FOCUs maps highlights the pervasiveness of constraints during developmentparticularly when building functional prototypes-and helps prioritize efforts to remove such constraints.

\section{CONCLUSION}

We believe the evolutionary and multiplyconstrained aspect of software design is captured well by the FOCUs model. The FOCUs approach is generalizable to a wide range of design and prototyping situations, but full assessments have yet to be performed. However, given its effectiveness with the smart scheduling problem, we are cautiously optimistic.

\section{REFERENCES}

1. Au, I. (1996). The Advising Workbench: A case study in the functional evolution of software. Unpublished master's thesis, Department of M. \& I. E., University of Illinois at Urbana-Champaign, Urbana, IL.

2. MacLean, A., Young, R., Bellotti, V., \& Moran, T. (1996) Questions, Options, and Criteria: Elements of Design Space Analysis. In T. P. Moran and J. M. Carroll (Eds.), Design Rationale: Concepts, techniques, and use (pp. 53-105). Hillsdale, NJ: Lawrence Erlbaum Associates.

3. Moran, T.P., \& Carroll, J.M. (1996). Overview of design rationale. In T.P. Moran and J.M. Carroll (Eds.), Design rationale: Concepts, techniques, and use (pp. 1-19). Hillsdale, NJ: Lawrence Erlbaum Associates, Inc.

4. Rasmussen, J., Pejtersen, A. \& Goodstein, L.P. (1994). Cognitive engineering: Concepts and applications. New York: Wiley.

5. Sanderson, P., Iozzo, N., Buberel, J., \& Au, I. (1995). The Advising Workbench: Participation-Based Development of a Software Environment to Support Student Advising. Proceedings of the 39th Annual Meeting of the Human Factors and Ergonomics Society (pp. 1180-1184). Santa Monica, CA: Human Factors and Ergonomics Society. 